\title{
Radiography nomography: Utility of a calculator in rational preoperative decision making for subsolid pulmonary nodules
}

\author{
Bryan M. Burt, MD \\ From the Division of General Thoracic Surgery, Department of Surgery, Baylor College of Medicine, Houston, \\ Tex. \\ Disclosures: Author has nothing to disclose with regard to commercial support. \\ Received for publication Oct 25, 2016; accepted for publication Oct 27, 2016; available ahead of print Nov 18, \\ 2016. \\ Address for reprints: Bryan M. Burt, MD, Division of General Thoracic Surgery, Department of Surgery, Baylor \\ College of Medicine, One Baylor Plaza, BCM 390, Houston, TX 77005 (E-mail: bryan.burt@ bcm.edu). \\ J Thorac Cardiovasc Surg 2017;153:460-1 \\ $0022-5223 / \$ 36.00$ \\ Copyright (C) 2016 by The American Association for Thoracic Surgery \\ http://dx.doi.org/10.1016/j.jtcvs.2016.10.047
}

The ground-glass opacity and the subsolid nodule are increasingly recognized radiographic representations of lung adenocarcinoma. The histology most commonly present in these radiographically defined lesions are adenocarcinomas of the lepidic histology spectrum, ranging from the preinvasive adenocarcinoma in situ to minimally invasive adenocarcinoma and to the more invasive lepidic predominant adenocarcinoma. ${ }^{1}$ A breadth of literature has demonstrated radiographic-pathologic associations among these interesting tumors with general correlation of radiographic ground glass to preinvasive histology and with general correlation of radiographic solid components to invasive pathology..$^{2-4}$ A clinical tool for the precise preoperative prediction of invasive histology has not been developed and could have important clinical utility.

In this issue of the Journal, Jin and colleagues ${ }^{5}$ have constructed and validated a nomogram with robust discrimination for calculating the risk of invasive pulmonary adenocarcinoma among a single-institution retrospective cohort of 273 patients who underwent resection of a peripheral subsolid lung nodule. A major advantage of a prediction tool such as this one is its implications for determining the extent of resection of radiographic subsolid pulmonary nodules, a proportion of which will contain invasive adenocarcinoma and a proportion of which will contain noninvasive or minimally invasive lesions. In this series, for example, $54 \%$ of subsolid nodules were pathologically invasive adenocarcinomas for which anatomic resection such as lobectomy should be offered, and the remainder were benign, preinvasive, or minimally invasive lesions for which sublobar resection could be considered. Whereas no randomized trials have evaluated the long-term oncologic outcomes of sublobar resection for these preinvasive and minimally invasive adenocarcinoma lesions, resection of these entities is associated with $100 \%$ disease-free survival (in studies including both lobectomy and sublobar

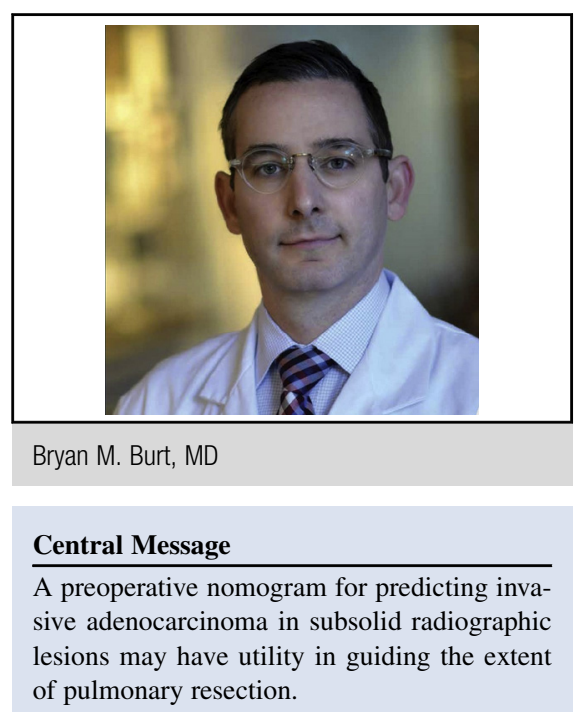

See Article page 462.

resections), ${ }^{1,6,7}$ and a number of retrospective studies have demonstrated excellent long-term survival outcomes with sublobar resection for adenocarcinomas that were predominantly ground glass radiographically. ${ }^{8,9}$ Supporting the rationale for this type of preoperative decision-making for subsolid nodules (lobectomy vs sublobar resection) is that preoperative needle biopsy and intraoperative frozen section generally cannot sufficiently distinguish invasive from preinvasive disease, secondary predominantly to sampling error. ${ }^{1,10}$ However, as the accuracy of frozen section for this disease improves, as it has in select centers, ${ }^{7}$ the clinic utility of such a nomogram will diminish.

In Jin and colleagues' study, ${ }^{5}$ the nomogram for predicting the probability of invasive adenocarcinoma was constructed from variables including computed tomography attenuation, vascular convergence, pleural tag, spiculation, lesion size, and solid proportion. Several of these variables, in other institutions and countries, are not universally reported features of cross-sectional chest imaging. Practical implementation of such a nomogram requires experienced chest radiologists participating in nomogram point assignment, and the generalizability of this test to other institutions and countries requires validation. Of note, this nomogram was constructed from a dataset composed of only surgically resected lesions, and it will be imperative 
to validate these methods among a larger cohort of individuals with subsolid pulmonary nodules treated both surgically and nonsurgically, ideally in a prospective trial.

\section{References}

1. Travis WD, Brambilla E, Noguchi M, Nicholson AG, Geisinger KR, Yatabe Y, et al. International Association for the Study of Lung Cancer/American Thoracic Society/European Respiratory Society International multidisciplinary classification of lung adenocarcinoma. J Thorac Oncol. 2011;6:244-85.

2. Takahashi M, Shigematsu Y, Ohta M, Tokumasu H, Matsukura T, Hirai T. Tumor invasiveness as defined by the newly proposed IASLC/ATS/ERS classification has prognostic significance for pathologic stage IA lung adenocarcinoma and can be predicted by radiologic parameters. J Thorac Cardiovasc Surg. 2014; 147:54-9.

3. Suzuki K, Koike T, Asakawa T, Kusumoto M, Asamura H, Nagai K, et al. A prospective radiological study of thin-section computed tomography to predict pathological noninvasiveness in peripheral clinical IA lung cancer (Japan Clinical Oncology Group 0201). J Thorac Oncol. 2011;6:751-6.

4. Austin JH, Garg K, Aberle D, Yankelevitz D, Kuriyama K, Lee HJ, et al, Radiologic implications of the 2011 classification of adenocarcinoma of the lung. Radiology. 2013;266:62-71.
5. Jin C, Cao J, Cai Y, Wang L, Liu K, Shen W, et al. A nomogram for predicting the risk of invasive pulmonary adenocarcinoma for patients with solitary peripheral sub-solid nodules. J Thorac Cardiovasc Surg. 2017;153:462-9.

6. Kadota K, Villena-Vargas J, Yoshizawa A, Motoi N, Sima CS, Riely GJ, et al. Prognostic significance of adenocarcinoma in situ, minimally invasive adenocarcinoma, and nonmucinous lepidic predominant invasive adenocarcinoma of the lung in patients with stage I disease. Am J Surg Pathol. 2014; 38:448-60.

7. Liu S, Wang R, Zhang Y, Li Y, Cheng C, Pan Y, et al. Precise diagnosis of intraoperative frozen section is an effective method to guide resection strategy for peripheral small-sized lung adenocarcinoma. J Clin Oncol. 2016; 34:307-13.

8. Cho JH, Choi YS, Kim J, Kim HK, Zo JI, Shim YM. Long-term outcomes of wedge resection for pulmonary ground-glass opacity nodules. Ann Thorac Surg. 2015;99:218-22.

9. Tsutani Y, Miyata Y, Nakayama H, Okumura S, Adachi S, Yoshimura M, et al. Appropriate sublobar resection choice for ground glass opacity-dominant clinical stage IA lung adenocarcinoma: wedge resection or segmentectomy. Chest 2014;145:66-71.

10. Yeh YC, Nitadori J, Kadota K, Yoshizawa A, Rekhtman N, Moreira AL, et al. Using frozen section to identify histological patterns in stage I lung adenocarcinoma of $\leq 3 \mathrm{~cm}$ : accuracy and interobserver agreement. Histopathology. 2015; 66:922-38. 